\title{
SUBBUNDLES OF THE TANGENT BUNDLE
}

\author{
BY
}

\section{R. E. STONG}

ABSTRACT. This paper studies pairs $(M, \xi)$ where $M$ is a closed manifold and $\xi$ is a $k$-dimensional subbundle of the tangent bundle of $M$ in terms of cobordism.

1. Introduction. The purpose of this note is to analyze pairs $(M, \xi)$ where $M$ is an $n$-dimensional manifold and $\xi$ is a $k$-dimensional subbundle of the tangent bundle of $M, k \leqslant n$, in terms of cobordism.

In $\S 2$, the cobordism class of $M$ is analyzed and the main result is

Proposition. A class $\alpha \in \mathfrak{N}_{n}$ is represented by a manifold $M^{n}$ whose tangent bundle has a $k$-dimensional subbundle, $k \leqslant n$, if and only if either

(a) $k$ is even, or

(b) $k$ is odd and $w_{n}(\alpha)=0$.

In section $\S 3$, the case $k=1$, i.e., $\xi$ a line bundle, will be studied more closely. One defines a homomorphism $\theta: \Re_{n}\left(B O_{1}\right) \rightarrow Z_{2}$ as follows. If $\alpha \in$ $\mathfrak{N}_{n}\left(B O_{1}\right)$, choose a manifold $M^{n}$ and map $f: M^{n} \rightarrow B O_{1}$ representing $\alpha$. Let $i \in H^{1}\left(B O_{1} ; Z_{2}\right)$ be the nonzero class, and let $\theta(\alpha)$ be the characteristic number

$$
\left\{w_{n}(M)+w_{n-1}(M) f^{*}(i)+\cdots+w_{n-r}(M)\left(f^{*}(i)\right)^{r}+\cdots+\left(f^{*}(i)\right)^{n}\right\}[M] .
$$

Letting $\gamma$ be the universal line bundle over $B O_{1}$, the class $\alpha$ is the class of the pair $\left(M, f^{*}(\gamma)\right)$, and interpreting $\Re_{n}\left(B O_{1}\right)$ as the cobordism classes of $n$-manifolds with a line bundle, one has

Proposition. A class $\alpha \in \mathfrak{N}_{n}\left(B O_{1}\right)$ is represented by a pair $\left(M^{n}, \xi\right)$ where $\xi$ is a sub-line-bundle of the tangent bundle of $M$ if and only if $\theta(\alpha)=0$.

Note. In order to make this result seem plausible, one should note that the given characteristic number is the $n$th Stiefel-Whitney number of $\tau_{M}-f^{*}(\gamma)$, which is an $(n-1)$-plane bundle if $f^{*}(\gamma)$ is a subbundle of $\tau_{M}$.

In $\S 4$, the problem is stabilized, and the main result is

Proposition. A class $\alpha=[M, f] \in \mathfrak{N}_{n}\left(B O_{k}\right)$ is represented by a pair $\left(M^{\prime}, \xi^{\prime}\right)$ with $\tau_{M^{\prime}} \oplus 1 \cong \xi^{\prime} \oplus \eta^{\prime} \oplus 1$ where $\eta^{\prime}$ is an $n-k$ plane bundle if 
and only if every Stiefel-Whitney number of $\alpha$ involving a class $w_{i}\left(\tau-f^{*}(\gamma)\right)$ for $i>n-k$ is zero.

In $\S 5$, the case $k=2$ is studied more closely.

The author is indebted to the National Science Foundation for financial support during this work.

2. The cobordism class of $M$.

LEMMA 2.1. If $M^{n}$ is a closed n-manifold and $\xi^{k}$ is a subbundle of the tangent bundle of $M$ with $k$ odd, then $w_{n}[M]=0$; i.e., $M$ has even Euler characteristic.

Proof. If $n$ is odd, $w_{n}[M]=0$, so one may assume $n$ even. Let $k=$ $2 p+1, n-k=2 q+1$ and let $\eta$ be a complement of $\xi$ in $\tau$, the tangent bundle of $M$, so that $\xi \oplus \eta=\tau$. Then

$$
\begin{aligned}
w_{n}[M] & =w_{n}(\tau)[M] \\
& =w_{2 p+1}(\xi) \cup w_{2 q+1}(\eta)[M], \\
& =\left(S q^{1} w_{2 p}(\xi)+w_{1}(\xi) \cup w_{2 p}(\xi)\right) \cup w_{2 q+1}(\eta)[M] \\
& =\left\{S q^{1} w_{2 p}(\xi) \cup w_{2 q+1}(\eta)+\left(w_{1}(\tau)+w_{1}(\eta)\right) \cup w_{2 p}(\xi) \cup w_{2 q+1}(\eta)\right\}[M] \\
& =\left\{S q^{1} w_{2 p}(\xi) \cup w_{2 q+1}(\eta)+v_{1}(\tau) \cup w_{2 p}(\xi) \cup w_{2 q+1}(\eta)\right. \\
& \left.\quad+w_{2 p}(\xi) \cup S q^{1} w_{2 q+1}(\eta)\right\}[M] \\
& =\left\{v_{1}(\tau) \cup+S q^{1}\right\}\left\{w_{2 p}(\xi) w_{2 q+1}(\eta)\right\}[M]
\end{aligned}
$$

but cup-product with the $\mathrm{Wu}$ class $v_{1}(\tau)=w_{1}(\tau)$ gives $S q^{1}$, and so this vanishes.

In order to prove the converse, one needs some examples of manifolds. For this, one may use the result of $[5,3.4]$ :

LEMMA 2.2. Let $R P\left(n_{1}, n_{2}, \cdots, n_{t}\right), t>1$, be the bundle of lines in the fibers of $\lambda_{1} \oplus \cdots \oplus \lambda_{t}$ over $R P\left(n_{1}\right) \times \cdots \times R P\left(n_{t}\right)$, where $\lambda_{i}$ is the pullback of the canonical bundle over $R P\left(n_{i}\right)$. Then $R P\left(n_{1}, \cdots, n_{t}\right)$ is a closed manifold of dimension $n+t-1$ where $n=n_{1}+\cdots+n_{t}$, and is indecomposable in $\mathfrak{N}_{*}$ if and only if

$$
\left(\begin{array}{c}
n+t-2 \\
n_{1}
\end{array}\right)+\cdots+\left(\begin{array}{c}
n+t-2 \\
n_{t}
\end{array}\right)
$$

is odd. 
One now defines manifolds $X^{n}$ of dimension $n$ for $n \neq 2^{s}-1$ and $n \neq 2$ as follows:

(a) if $n=4 s, s \geqslant 1$,

$$
X^{n}=R P(\underbrace{1, \cdots, 1}_{2 s}, 0)
$$

(b) if $n=4 s+2, s \geqslant 1$,

$$
X^{n}=R P(\underbrace{1, \cdots, 1}_{2 s}, 0,0,0),
$$

(c) if $n=2^{p}(2 q+1)-1, p, q>0$,

$$
X^{n}=R P(2^{p}, \underbrace{1, \cdots, 1}_{2^{p_{q-1}}}, 0) .
$$

The above criterion immediately shows that these manifolds are indecomposable in $\mathfrak{N}_{*}$.

The manifolds $X^{n}$ have the additional property that, for each integer $k \leqslant n$, the tangent bundle of $X^{n}$ has a $k$-dimensional subbundle. In fact, for $n \neq 5$, the tangent bundle of $X^{n}$ is a Whitney sum of line bundles.

To see this, let $\lambda$ be the canonical line bundle over $R P\left(n_{1}, \cdots, n_{t}\right)$ and $\pi: R P\left(n_{1}, \cdots, n_{t}\right) \rightarrow R P\left(n_{1}\right) \times \cdots \times R P\left(n_{t}\right)$ the projection. Let $\lambda_{i}$ denote $\pi^{*}\left(\lambda_{i}\right)$ and $\tau_{i}$ the pullback of the tangent bundle of $R P\left(n_{i}\right)$. Then

$$
\tau_{R P\left(n_{1}, \cdots, n_{t}\right)} \cong \pi^{*} \tau_{R P\left(n_{1}\right) \times \cdots \times R P\left(n_{t}\right)} \oplus \mu \cong \tau_{1} \oplus \cdots \oplus \tau_{t} \oplus \mu
$$

where $\mu$ is the bundle along the fibers. Then

$$
\mu \oplus l \cong\left(\lambda \otimes \lambda_{1}\right) \oplus \cdots \oplus\left(\lambda \otimes \lambda_{t}\right) \quad \text { and } \quad \tau_{i} \oplus l=\left(n_{i}+1\right) \lambda_{i}
$$

where $l$ is the trivial line bundle. If $n_{i}=0$ or $1, \tau_{i}$ is trivial, since the tangent bundles of $R P(1)=S^{1}$ and $R P(0)=$ point are trivial. Adding the trivial $\tau_{i}$ with $n_{i}=1$ to other $\tau_{j}$ or $\mu$ represents them as sums of line bundles.

For $n=5, R P(2,1,0)$ has tangent bundle $\tau_{1} \oplus l \oplus \mu$ which is a line bundle and two 2-plane bundles, while in all other cases there are at least two $l$ s and the tangent bundle is a sum of line bundles.

One now has

Proposition 2.3. A class $\alpha \in \mathfrak{N}_{n}$ is represented by a manifold $M^{n}$ whose tangent bundle has a $k$-dimensional subbundle, $k \leqslant n$, if either:

(a) $k$ is even, or

(b) $k$ is odd and $w_{n}(\alpha)=0$. 
Proof. Every class $\alpha \in \mathfrak{R}_{n}$ is represented by the disjoint union of manifolds

$$
\underbrace{R P(2) \times \cdots \times R P(2)}_{q} \times X^{n_{1}} \times \cdots \times X^{n_{s}}
$$

with $2 q+n_{1}+\cdots+n_{s}=n$. For any integer $k \leqslant n$ of the form $2 u+v$ with $u \leqslant q, v \leqslant n_{1}+\cdots+n_{s}$, this component has a subbundle of its tangent bundle of dimension $k$. In particular, every even integer can be put in this form, and every odd integer will be of this form except for the component $[R P(2)]^{n / 2}$ which has $w_{n} \neq 0$.

This completes the proof of the proposition given in the introduction.

REMARK. If $\xi$ is the line bundle over $R P(1)$ and $\lambda$ is the line bundle over the Klein bottle $R P(\xi \oplus l)$, then the 5-manifold $R P(\lambda \oplus 3)$ is indecomposable in $\mathfrak{R}_{*}$ and has tangent bundle a sum of line bundles. This manifold could be used in place of $X^{5}$ and so five plays no special role.

\section{Line bundles.}

LEMMA 3.1. If $M^{n}$ is a closed n-manifold, $\xi$ a sub-line-bundle of the tangent bundle of $M$ and $f: M \rightarrow B O_{1}$ classifies $\xi$, then $\theta([M, f])=0$.

Proof. Let $\eta$ be a complement in $\tau$ for $\xi$. Then $w(\eta)=w(\tau) / w(\xi)$, so since $\eta$ is an $(n-1)$-plane bundle

$$
0=w_{n}(\eta)=w_{n}(\tau)+w_{n-1}(\tau) w_{1}(\xi)+\cdots+\left(w_{1}(\xi)\right)^{n} .
$$

Since $w_{1}(\xi)=f^{*}(i)$ and $w_{i}(\tau)=w_{i}(M)$, this gives $\theta([M, f])=0$.

In order to prove the converse, one needs to analyze the bordism of $B O_{1}$. Henceforth, classes of $\Re_{*}\left(B O_{1}\right)$ will be denoted $[M, \xi]$ where $M$ is a closed manifold and $\xi$ is a line bundle over $M$. There is a homomorphism of $\mathfrak{N}_{*}$ modules, called the Smith homomorphism,

$$
\Delta: \mathfrak{N}_{*}\left(B O_{1}\right) \rightarrow \mathfrak{N}_{*}\left(B O_{1}\right)
$$

of degree -1 assigning to $[M, \xi]$ the class $[N, \xi \mid N]$ where $N \subset M$ is the codimension one submanifold of $M$ dual to $\xi$. Specifically, if $f: M \rightarrow B O_{1}=$ $R P(\infty)$ classifies $\xi, f$ maps $M$ into some $R P(n)$ and may be homotoped in $R P(n)$ to be transverse regular on $R P(n-1)$, with $N$ then taken to be the inverse image of $R P(n-1)$.

Letting $1=$ [point, $l] \in \mathfrak{R}_{0}\left(B O_{1}\right)$, there are unique classes $x_{i}=\left[M^{i}, \xi^{i}\right]$ $\in \mathfrak{N}_{i}\left(B O_{1}\right), i \geqslant 0$, with

(1) $x_{0}=1$,

(2) $\Delta x_{i}=x_{i-1}$, and

(3) for $i>0, M^{i}$ bounds. 
These classes form a base for $\mathfrak{N}_{*}\left(B O_{1}\right)$ as $\mathfrak{N}_{*}$ module. (A proof of this statement, or more precisely, its complex analogue appears in $[2,(5.3)]$.

Lemma 3.2. For $i>0, x_{i}$ is the class of the canonical bundle $\lambda$ over $R P(1,0, \cdots, 0)(i-10$ 's).

Proof. In $[1,(2.2)], R P(1,0, \cdots, 0)(i-10$ 's $)$ is denoted $R P(\xi \oplus(i-1))$, where $\xi$ is the canonical line bundle over $R P(1)$, and is shown to bound. In $[4$, p. 160] it is shown that for any vector bundle $\rho$ over $M$, the submanifold dual to $\lambda$ over $R P(\rho \oplus l)$ is $R P(\rho)$, from which the behaviour of $\Delta$ follows.

For $i>1$, the tangent bundle of $R P(1,0, \cdots, 0)(i-10$ 's) is $1 \oplus \mu=$ $\lambda \otimes \pi^{*}(\xi) \oplus(i-1) \lambda$, which contains a copy of $\lambda$, so $\theta\left(x_{i}\right)=0$ if $i>1$.

Now if $\xi$ is a line bundle over $M$, and $N$ is a closed manifold, $\pi_{M}^{*}(\xi)$ is a line bundle over $M \times N$, with $[N] \cdot[M, \xi]=\left[M \times N, \pi_{M}^{*}(\xi)\right]$ giving the module structure of $\mathfrak{N}_{*}\left(B O_{1}\right)$. If $N$ has dimension $n$, it is immediate that $\theta([N] \cdot[M, \xi])=w_{n}[N] \cdot \theta([M, \xi])$.

Since $\theta\left(x_{0}\right)=\theta\left(x_{1}\right)=1$, one then has

LEMMA 3.3. $\theta\left(\Sigma_{i}\left[N^{n-i}\right] x_{i}\right)=w_{n}\left(N^{n}\right)+w_{n-1}\left(N^{n-1}\right)$.

Proposition 3.4. If $\alpha \in \mathfrak{N}_{n}\left(B O_{1}\right)$ with $\theta(\alpha)=0$, then $\alpha=[M, \xi]$ where $\xi$ is a sub-line-bundle of the tangent bundle of $M$.

PROOF. Let $\alpha=\sum_{i=1}^{n} a_{i} x_{i}$ with $a_{i} \in \mathfrak{N}_{n-i}$. Then $w_{n}\left(a_{0}\right)=0, w_{n-1}\left(a_{1}\right)=0$, for if $n$ is odd $w_{n}\left(a_{0}\right)=0$ for dimensional reasons while $w_{n-1}\left(a_{1}\right)=\theta(\alpha)=0$ and if $n$ is even $w_{n-1}\left(a_{1}\right)=0$ for dimensional reasons while $w_{n}\left(a_{0}\right)=\theta(\alpha)=0$. By $[1,(4.5)]$ there are manifolds $N^{n}$ and $N^{n-1}$ fibered over $S^{1}$, with $\left[N^{n-i}\right]=$ $a_{i}, i=0,1$. Choose manifolds $N^{n-i}$ representing $a_{i}$ for $i>1$, and let

$$
M^{n}=N^{n} \cup\left(N^{n-1} \times R P(1)\right) \cup \bigcup_{i>1}\left(N^{n-i} \times R P(1, \underbrace{0, \cdots, 0}_{i-1})\right)
$$

and let $\xi$ be the line bundle over $M$ whose restriction to $N^{n}$ is trivial, to $N^{n-1} \times R P(1)$ is the pullback of the canonical bundle over $R P(1)$ and to $N^{n-i} \times R P(1,0, \cdots, 0)$ is the pullback of $\lambda$. Then $\alpha=[M, \xi]$.

Since $N^{n}$ fibers over $S^{1}$, the pullback of $\tau_{S^{1}}$ is a trivial line bundle in $\tau_{N^{n}}$. Since $N^{n-1} \times R P(1)$ fibers over $S^{1} \times S^{1}$, its tangent bundle contains a trivial 2 plane-bundle, but if $\xi^{\prime}$ is the canonical bundle over $R P(1), 2 \xi^{\prime}=2$ so the tangent bundle contains two copies of the pullback of $\xi^{\prime}$. As noted, $\lambda$ is a subbundle of the tangent bundle of $R P(1,0, \cdots, 0)(i-10$ 's) if $i>1$.

Thus $\xi$ is a subbundle of the tangent bundle of $M$.

Combining this with Lemma 3.1 gives the second proposition of the introduction. 
Now restricting attention to oriented manifolds one has

Proposition 3.5. A class $\alpha \in \Omega_{n}$ is represented by an oriented manifold $M^{n}$ whose tangent bundle contains a line bundle if and only if the Stiefel-Whitney number $w_{n}(\alpha)$ is zero.

A class $\alpha \in \Omega_{n}(R P(\infty))$ is represented by a pair $\left[M^{n}, \xi\right]$ where $\xi$ is a sub-line-bundle of the tangent bundle of the oriented manifold $M$ if and only if the Stiefel-Whitney number $\theta(\alpha)$ is zero.

PROof. These conditions are clearly necessary. To see that they are sufficient, consider $\alpha \in \Omega_{n}$ for which $w_{n}(\alpha)=0$ and choose a representative $M^{n}$ for $\alpha$. Using surgery, one may replace $M$ by the connected sum of its components; i.e., may assume $M$ connected. If $n$ is odd, the tangent bundle has a nonvanishing section, while if $n$ is even, such a section exists if and only if the Euler class of the tangent bundle $X(\tau)$ is zero. Since $M$ is connected, $X(\tau)=$ $\chi(M) \sigma$, where $\chi(M)$ is the Euler characteristic of $M$ and $\sigma$ is a generator of $H^{n}(M ; Z) \cong Z$. Mod 2, $\chi(M)$ is $w_{n}(\alpha)$ so $\chi(M)$ is even, and by forming the connected sum of $M$ with copies of $S^{p} \times S^{q}$ for suitable $p, q>0$, one obtains a new $M$ with $\chi(M)=0$ also in $\alpha$. [Note. If $n=2, \alpha=0$ and $M$ may be taken empty or $S^{1} \times S^{1}$ while if $n=2 k, k>1$, the connected sum with $S^{2} \times S^{n-2}$ increases $\chi$ by 2 while that with $S^{1} \times S^{n-1}$ decreases it by 2.] Thus every $\alpha \in \Omega_{n}$ with $w_{n}(\alpha)=0$ is represented by a manifold $M^{n}$ for which $\tau_{M}$ contains a trivial line bundle.

Now turning to $\Omega_{*}(R P(\infty))$, one has $\Omega_{*}(R P(\infty)) \cong \Omega_{*} \oplus \widetilde{\Omega}_{*}(R P(\infty))$ and $\widetilde{\Omega}_{*}(R P(\infty)) \cong \mathfrak{N}_{*-1}$. A class in the $\Omega_{n}$ summand of $\Omega_{n}(R P(\infty))$ is represented by a manifold $M^{n}$ with trivial line bundle, and $\theta([M, 1])=\left\langle w_{n}(\tau),[M]\right\rangle$ so that by the above, a class $\alpha$ in the $\Omega_{*}$ summand is represented by a subbundle if and only if $\theta(\alpha)=0$. The summand $\Re_{n-1}$ of $\Omega_{n}(R P(\infty))$ is realized as follows. If $\beta \in \mathfrak{N}_{n-1}$, let $N^{n-1}$ be a manifold in $\beta$ and let $M^{n}$ be the real projective space bundle $R P(\xi \oplus 1)$ where $\xi$ is the determinant bundle of the tangent bundle of $N$ and let $\lambda$ be the canonical line bundle over $R P(\xi \oplus 1)$. Assigning to $\beta$ the class of $[M, \lambda]$ gives the isomorphism $\mathfrak{N}_{n-1} \cong \widetilde{\Omega}_{n}(R P(\infty))$. Now $\theta([M, \lambda])=w_{n-1}(\beta)$, and if $\theta([M, \lambda])=0, \beta$ is represented by a manifold $N$ whose tangent bundle has a section and so $\lambda$ is a subbundle of the tangent bundle of $R P(\xi \oplus 1)$. Noting that $\theta$ vanishes on the $\Omega_{*}$ summand if $n$ is odd and on the $\widetilde{\Omega}_{*}(R P(\infty))$ summand if $n$ is even, one sees that every class in the kernel of $\theta$ is realized by a subbundle of the tangent bundle.

4. Stabilization. One now considers stabilization of the subbundle problem. This permits the use of homotopy theoretic techniques.

One may consider a manifold $M^{n}$ together with an isomorphism $\tau_{M} \oplus$ 
$j \cong \xi^{k} \oplus \eta^{n-k} \oplus j$ where $j$ denotes a trivial $j$ plane bundle. By stability the existence of an isomorphism is independent of $j$ if $j \geqslant 1$. The manifold $M^{n}$ with this structure bounds if $M=\partial V$ where $\tau_{V} \oplus(j-1) \cong \rho^{k} \oplus \sigma^{n-k+1} \oplus$ $(j-1)$ is a compatible decomposition; i.e., $\rho$ restricts to $\xi$ and $\sigma$ to $\eta \oplus 1$. Assuming $V$ has no closed components, $V$ has the homotopy type of an $n$-dimensional complex, so $\tau_{V} \cong \rho \oplus \sigma$, but this need not be compatible with the chosen isomorphism along $M$ unless $j>1$.

Let $\phi_{r}^{k}: B O_{k} \times B O_{r} \rightarrow B O$ be a map classifying the complement of the Whitney sum $\gamma_{k} \oplus \gamma_{r}$ of the universal bundles (converted to a fibration). The structure on $M$ is precisely a lift of the normal map of $M$ to $B O_{k} \times B O_{n-k}$, while that of $V$ is a lift to $B O_{k} \times B O_{n-k+1}$.

The techniques of bordism of manifolds with normal structure [3] give that the bordism group of manifolds $M^{n}$ of the gi 'en type is the image of the stable homotopy homomorphism

$$
\pi_{n}^{S}\left(T\left(B O_{k} \times B O_{n-k}\right)\right) \rightarrow \pi_{n}^{S}\left(T\left(B O_{k} \times B O_{n-k+1}\right)\right)
$$

where $T\left(B O_{k} \times B O_{r}\right)$ is the Thom spectrum associated with the fibration $\phi_{r}^{k}$.

Specifically, if one takes the induced fibration

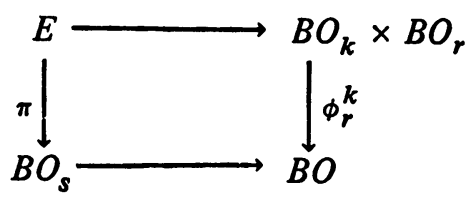

then $\pi_{n}^{S}\left(T\left(B O_{k} \times B O_{r}\right)\right)=\lim _{s \rightarrow \infty} \pi_{n+s}\left(T\left(\pi^{*}\left(\gamma_{s}\right)\right)\right)$. One may also describe these groups as

$$
\pi_{n}^{S}\left(T\left(B O_{k} \times B O_{r}\right)\right)=\lim _{s, t \rightarrow \infty} \pi_{n+s+t}\left(T\left(\gamma_{s} \oplus \gamma_{t}\right)\right)
$$

where $\gamma_{s}, \gamma_{t}$ are the universal $s$ and $t$ plane bundles over the Grassmann manifolds $G_{k, s}$ and $G_{r, t}$.

One may now consider the homomorphism

$$
\begin{gathered}
\pi_{n}^{S}\left(T\left(B O_{k} \times B O_{n-k}\right)\right) \rightarrow \lim _{r \rightarrow \infty} \pi_{n}^{S}\left(T\left(B O_{k} \times B O_{r}\right)\right) \\
\| \\
\pi_{n}^{S}\left(T\left(B O_{k} \times B O\right)\right) .
\end{gathered}
$$

One has $\pi_{1} \times \oplus: B O_{k} \times B O \rightarrow B O_{k} \times B O$, which is a homotopy equivalence, and induces an equivalence $T\left(B O_{k} \times B O\right) \cong B O_{k}^{+} \wedge M O$ and hence

$$
\pi_{n}^{S}\left(T\left(B O_{k} \times B O\right)\right) \cong \Re_{n}\left(B O_{k}\right) .
$$


This describes the forgetful homomorphism assigning to $M^{n}$ with its structure the bordism class of $(M, \xi)$.

One now embarks on a program of analyzing the stable homotopy groups involved.

LEMMA 4.1. Let $\gamma_{s}$ be the universal s plane bundle over $G_{r, s}, s>r$, and let $p$ be an odd prime. Then $\tilde{H}^{i}\left(T\left(\gamma_{s}\right) ; Z_{p}\right)=0$ for $i<r+s$.

Proof. One has the inclusion $G_{r, s} \subset G_{r+1, s}$ with $G_{r+1, s}$ obtained by attaching cells of dimension $(r+1)$ and higher. This induces an inclusion of Thom spaces $T\left(\gamma_{s} \mid G_{r, s}\right) \subset T\left(\gamma_{s} \mid G_{r+1, s}\right)$ and the cofiber has cells of dimension $r+1+s$ and higher. From the exact cohomology sequence

$$
\widetilde{H}^{i}\left(T\left(\gamma_{s} \mid G_{r, s}\right) ; Z_{p}\right) \cong \widetilde{H}^{i}\left(T\left(\gamma_{s} \mid G_{r+1, s}\right) ; Z_{p}\right) \text { if } i<r+s .
$$

Thus

$$
\widetilde{H}^{i}\left(T\left(\gamma_{s} \mid G_{r, s}\right) ; Z_{p}\right) \cong \widetilde{H}^{i}\left(T\left(\gamma_{s} \mid G_{r+t, s}\right) ; Z_{p}\right) \text { if } i<r+s, t \geqslant 0,
$$

but for $t$ large this is $\tilde{H}^{i}\left(M O_{s} ; Z_{p}\right)$ which is zero.

LEMMA 4.2. $\pi_{i}^{S}\left(T\left(B O_{k} \times B O_{r}\right)\right)$ is a 2 group if $i<k+r$.

Proof. Let $\gamma_{s}, \gamma_{t}$ be the universal bundles over $G_{k, s}$ and $G_{r, t}, s$ and $t$ large. Then $T\left(\gamma_{s} \oplus \gamma_{t}\right)=T\left(\gamma_{s}\right) \wedge T\left(\gamma_{t}\right)$ and $\widetilde{H}^{i}\left(T\left(\gamma_{s} \oplus \gamma_{t}\right) ; Z_{p}\right)=0$ if $i<k+r+s+t$ if $p$ is odd. By the $\bmod C$ Hurewicz theorem $\pi_{i}\left(T\left(\gamma_{s} \oplus \gamma_{t}\right)\right)$ is a 2 group if $i<k+r+s+t$.

Thus, for $r \geqslant n-k+1, \pi_{n}^{S}\left(T\left(B O_{k} \times B O_{r}\right)\right)$ is a 2 group, and the problem is entirely a 2 primary problem.

In order to begin the 2 primary analysis, one analyzes a cofibration of spectra

$$
T\left(B O_{k} \times B O_{r}\right) \rightarrow T\left(B O_{k} \times B O_{r+1}\right) \rightarrow \mathrm{X}
$$

which one realizes by a cofibration $T\left(\gamma_{s} \oplus \gamma_{t}\right) \rightarrow T\left(\gamma_{s} \oplus \gamma_{t}^{\prime}\right) \rightarrow X$ where $\gamma_{s}$, $\gamma_{t}$ are universal bundles over $G_{k, s}, G_{r, t}$ and $\gamma_{t}^{\prime}$ is the universal bundle over $G_{r+1, t}$, with $s$ and $t$ being large.

First, consider $G_{r+1, t}$ as the space of $r+1$ planes in $R^{r+1+t}$ with $\pi: D\left(\gamma_{r+1}\right) \rightarrow G_{r+1, t}$ the projection of the disc bundle. Letting $S\left(\gamma_{r+1}\right)$ be the unit sphere bundle, one has a cofibration

$$
\begin{array}{ccc}
\frac{D\left(\pi^{*}\left(\gamma_{t}^{\prime}\right) \mid S\left(\gamma_{r+1}\right)\right)}{S\left(\pi^{*}\left(\gamma_{t}^{\prime}\right) \mid S\left(\gamma_{r+1}\right)\right)} \rightarrow & \frac{D\left(\pi^{*}\left(\gamma_{t}^{\prime}\right)\right)}{S\left(\pi^{*}\left(\gamma_{t}^{\prime}\right)\right)} \rightarrow & \frac{D\left(\pi^{*}\left(\gamma_{t}^{\prime}\right)\right)}{D\left(\pi^{*}\left(\gamma_{t}^{\prime}\right) \mid S\left(\gamma_{r+1}\right)\right) \cup S\left(\pi^{*}\left(\gamma_{t}^{\prime}\right)\right)} . \\
\| & \| & \| \\
A & B & C
\end{array}
$$


Since $D\left(\pi^{*}\left(\gamma_{t}^{\prime}\right)\right)$ is identifiable with $D\left(\gamma_{r+1} \oplus \gamma_{t}^{\prime}\right), C$ is the Thom space of the trivial bundle $\gamma_{r+1}, \oplus \gamma_{t}^{\prime}$, and $C \cong \Sigma^{r+t+1}\left(G_{r+1, t}\right)$ is the $(r+t+1)$-fold suspension of $G_{r+1, t}$ with a base point adjoined. Since $\pi$ is a homotopy equivalence, $B \cong T\left(\gamma_{t}^{\prime}\right)$.

Finally, $S\left(\gamma_{r+1}\right)$ may be considered as pairs $(\alpha, x)$ with $\alpha$ an $(r+1)$ plane in $R^{r+1+t}$ and $x$ a unit vector in $\alpha$. Assigning to $(\alpha, x)$ the point $x \in S^{r+t}$ defines a fibration $p: S\left(\gamma_{r+1}\right) \rightarrow S^{r+t}$. The inverse image of $x \in S^{r+t}$ is the space of $r$ planes in $R^{r+1+t}$ orthogonal to $x$, i.e., $S\left(\gamma_{r+1}\right)$ is the Grassmann bundle of $r$ planes in the fibers of the tangent bundle of $S^{r+t}$. The inclusion $G_{r, t} \rightarrow G_{r+1, t}$ may then be considered as factoring via the inclusion as a fiber in $S\left(\gamma_{r+1}\right)$. The inclusion of the fiber $G_{r, t} \rightarrow S\left(\gamma_{r+1}\right)$ induces isomorphisms in homotopy and homology in dimensions less than $r+t-1$, and so the inclusion $T\left(\gamma_{t}\right) \rightarrow A$ is a homotopy equivalence (for the prime 2 ) in dimensions less than $r+2 t-1$. Since $t$ is large, one then obtains a cofibration

$$
T\left(\gamma_{t}\right) \rightarrow T\left(\gamma_{t}^{\prime}\right) \rightarrow \Sigma^{r+t+1}\left(G_{r+1, t}^{+}\right) .
$$

Smashing with $T\left(\gamma_{s}\right)$ gives a cofibration sequence

$$
T\left(\gamma_{s} \oplus \gamma_{t}\right) \rightarrow T\left(\gamma_{s} \oplus \gamma_{t}^{\prime}\right) \rightarrow T\left(\gamma_{s}\right) \wedge \Sigma^{r+t+1}\left(G_{r+1, t}\right)
$$

(i.e., $X$ may be identified with $T\left(\gamma_{s}\right) \wedge \Sigma^{r+t+1}\left(G_{r+1, t}^{+}\right)$for the prime 2, having isomorphic mod 2 cohomology up to dimension $s+r+2 t-1$ induced by a map of spaces).

One now considers $T\left(\gamma_{s}\right) \wedge \Sigma^{r+t+1}\left(G_{r+1, t}\right)$ as $\Sigma^{r+t+1} T\left(\gamma_{s}\right) \wedge G_{r+1, t}$ and analyzes the maps

$$
G_{k, s} \rightarrow G_{k, s+r+t+1} \rightarrow G_{m, s+r+t+1}
$$

inducing

$$
\Sigma^{r+t+1} T\left(\gamma_{s}\right) \rightarrow T\left(\gamma_{s+r+t+1}\right) \rightarrow M O_{s+r+t+1}
$$

( $m$ being large). The maps of Grassmannians induce isomorphisms in mod 2 cohomology in dimensions less than or equal to $k$ and hence the Thom spaces have isomorphic mod 2 cohomology in dimensions less than or equal to $k+s+r+t+1$.

Thus $X$ may be identified with $M O_{s+r+t+1} \wedge\left(G_{r+1, t}\right)^{+}$in dimensions less than or equal to $k+s+r+t+1$ (in mod 2 cohomology). In particular, in dimensions less than or equal to $k+s+r+t+1 \widetilde{H}^{*}\left(X ; Z_{2}\right)$ is a free module over the Steenrod algebra and

$$
\pi_{i+s+t}(X) \cong \pi_{i+s+t}\left(M O_{s+r+t+1} \wedge\left(G_{r+1, t}\right)^{+}\right) \cong \mathfrak{N}_{i-r-1}\left(G_{r+1, t}\right)
$$


if $i+s+t \leqslant k+s+r+t, i \leqslant k+r$ (for 2 primary structure).

Being given a manifold $M^{i}$ with $\tau_{M} \oplus j \cong \xi^{k} \oplus \eta^{r+1} \oplus(i+j-k-r-1)$ representing a class in $\pi_{i}^{S}\left(T\left(B O_{k} \times B O_{r+1}\right)\right), i \leqslant k+r$, the class in $\pi_{i}^{S}(\mathrm{X}) \cong$ $\mathfrak{N}_{i-r-1}\left(B O_{r+1}\right)$ obtained from the cofibration is represented by the submanifold of $M^{i}$ dual to $\eta^{r+1}$ with the $(r+1)$-plane bundle obtained by restricting $\eta$. The map to $\mathbf{X}$ is induced by including $T\left(\gamma_{t}^{\prime}\right)$ in $T\left(\gamma_{t}^{\prime} \oplus \gamma_{r+1}\right)$ and making the maps transverse regular involves finding the submanifold dual to $\gamma_{r+1}$, from which one has the given assertion.

On the other hand, a class in $\pi_{i}^{S}\left(T\left(B O_{k} \times B O_{r+1}\right)\right), i \leqslant k+r$, is in the image of $\pi_{i}^{S}\left(T\left(B O_{k} \times B O_{r}\right)\right)$ if and only if it goes to zero in $\pi_{i}^{S}(\mathrm{X})$. Since $\widetilde{H}^{*}\left(X ; Z_{2}\right)$ is a free module over the Steenrod algebra in dimensions up to $k+s+r+t+1$, a homotopy element in $\pi_{i+s+t}(X)$ is detected by $\bmod 2$ cohomology. Since $T\left(\gamma_{s} \oplus \gamma_{t}^{\prime}\right) \rightarrow X$ maps $\widetilde{H}^{*}\left(X ; Z_{2}\right)$ isomorphically onto the multiples of $\Phi\left(w_{r+1}\right)$, the Thom isomorphism image of $w_{r+1}$, in the $H^{*}\left(G_{k, s} \times G_{r+1, t} ; Z_{2}\right)$ module structure, this asserts that all characteristic numbers involving $w_{r+1}$ should vanish. Thus, one has

LEMMA 4.3. A manifold $M^{i}$ with $\tau_{M} \oplus j \cong \xi^{k} \oplus \eta^{r+1} \oplus(i+j-k-r-1)$ representing a class in $\pi_{i}^{S}\left(T\left(B O_{k} \times B O_{r+1}\right)\right), i \leqslant k+r$, comes from $\pi_{i}^{S}\left(T\left(B O_{k} \times B O_{r}\right)\right)$ if and only if all characteristic numbers involving $w_{r+1}(\eta)$ are zero.

For $r \geqslant n-k$, this determines the image of

$$
\pi_{n}^{S}\left(T\left(B O_{k} \times B O_{r}\right)\right) \rightarrow \pi_{n}^{S}\left(T\left(B O_{k} \times B O_{r+1}\right)\right)
$$

For $r \geqslant n-k+1$, this homomorphism is monic, which may be seen as follows. Consider the homomorphism

$$
\pi_{n+1}^{S}\left(T\left(B O_{k} \times B O_{r+1}\right)\right) \rightarrow \pi_{n+1}^{S}(\mathbf{X}) \text {. }
$$

Now $\pi_{n+1}^{S}(\mathrm{X}) \cong \mathfrak{N}_{n-r}\left(B O_{r+1}\right)$ for $n+1 \leqslant k+r$, and $\mathfrak{N}_{n-r}\left(B O_{r+1}\right)$ is generated over $Z_{2}$ by the manifolds

$$
P=M^{m} \times R P\left(\lambda_{1} \oplus k_{1}\right) \times \cdots \times R P\left(\lambda_{s} \oplus k_{s}\right) \times \text { (point) }
$$

where $\lambda_{i}$ is the nontrivial bundle over $R P(1), k_{i} \geqslant 0$, with $m+\left(k_{1}+1\right)+$ $\cdots+\left(k_{s}+1\right)=n-r$ with bundle

$$
\lambda^{(1)} \oplus \cdots \oplus \lambda^{(s)} \oplus(r+1-s)
$$

where $\lambda^{(i)}$ is the canonical bundle over $R P\left(\lambda_{i} \oplus k_{i}\right)$. To see this, one notes that the $R P(\lambda \oplus k), k \geqslant 0$, and the point generate $\mathfrak{N}_{*}\left(B O_{1}\right)$, over $\mathfrak{N}_{*}$ and forming the products of $r+1$ of these gives a $\mathfrak{N}_{*}$ generating set for $\mathfrak{N}_{*}\left(B O_{r+1}\right)$. 
One then considers the manifold

$$
Q=M^{m} \times R P\left(\lambda_{1} \oplus k_{1} \oplus 1\right) \times \cdots \times R P\left(\lambda_{s} \oplus k_{s} \oplus 1\right) \times R P(r+1-s)
$$

of dimension $m+\left(k_{1}+2\right)+\cdots+\left(k_{s}+2\right)+r+1-s=n-r+s+r+$ $1-s=n+1$ and letting $\lambda$ be the canonical line bundle over $R P(r+1-s)$, the submanifold dual to $\lambda^{(1)} \oplus \cdots \oplus \lambda^{(s)} \oplus(r+1-s) \lambda=\eta^{r+1}$ is the manifold $P$ given above, with $\eta$ restricting to the given bundle. Now the tangent bundle of $R P\left(\lambda_{i} \oplus k_{i} \oplus 1\right)$ is $\lambda^{(i)} \otimes \lambda_{i} \oplus\left(k_{i}+1\right) \lambda^{(i)}$ so

$$
\begin{aligned}
\tau_{Q} \oplus 1 & \cong\left[\tau_{M} \oplus\left(\lambda^{(1)} \oplus \lambda_{i} \oplus k_{i} \lambda^{(1)}\right) \oplus \cdots\right. \\
& \left.\left.\oplus \lambda^{(s)} \otimes \lambda_{s} \oplus k_{s} \lambda^{(s)}\right) \oplus \lambda\right] \oplus \eta \\
& =\xi^{\prime} \oplus \eta
\end{aligned}
$$

where $\xi^{\prime}$ is an $m+\left(k_{1}+1\right)+\cdots+\left(k_{s}+1\right)+1=n-r+1 \leqslant k$ bundle. Thus $\tau_{Q} \oplus 1 \oplus(k+r-n-1) \cong\left[\xi^{\prime} \oplus(k+r-1)\right] \oplus \eta=\xi^{k} \oplus \eta$ giving a structure on $Q$ mapping to the class of $P$ in $\mathfrak{N}_{n-r}\left(B O_{r+1}\right)$.

This proves that the forgetful homomorphism $\pi_{n}^{S}\left(T\left(B O_{k} \times B O_{r}\right)\right) \rightarrow$ $\mathfrak{N}_{n}\left(B O_{k}\right)$ is monic for $r \geqslant n-k+1$, and that

$$
\operatorname{im}\left\{\pi_{n}^{S}\left(T\left(B O_{k} \times B O_{n-k}\right)\right) \rightarrow \pi_{n}^{S}\left(T\left(B O_{k} \times B O_{n-k+1}\right)\right)\right\}
$$

is mapped monomorphically into $\mathfrak{N}_{n}\left(B O_{k}\right)$ with image precisely those classes for which all numbers involving $w_{i}\left(\tau-f^{*}\left(\gamma_{k}\right)\right)$ for $i>n-k$ are zero, or one has

Proposition 4.4. A class $\alpha=[M, f] \in \mathfrak{N}_{n}\left(B O_{k}\right)$ is represented by a manifold $M^{n}$ with $\tau_{M} \oplus 1 \cong f^{*}\left(\gamma_{k}\right) \oplus \eta^{n-k} \oplus 1$ if and only if all StiefelWhitney numbers of $\alpha$ involving $w_{i}\left(\tau-f^{*}\left(\gamma_{k}\right)\right)$ for $i>n-k$ are zero.

5. Two plane bundles. The purpose of this section is to prove

Proposition 5.1. A class $\alpha=[M, f] \in \mathfrak{N}_{n}\left(\mathrm{BO}_{2}\right)$ is represented by a pair $[M, f]$ with $f^{*}\left(\gamma_{2}\right)$ a subbundle of the tangent bundle of $M$ if and only if all characteristic numbers of $\alpha$ involving $w_{i}\left(\tau-f^{*}\left(\gamma_{2}\right)\right)$ with $i>n-2$ are zero.

To begin the proof, one wants manifolds $M_{i, j}$ of dimension $i+2 j$ for each $(i, j)$ and 2 plane bundles $\lambda_{i, j}$ over $M_{i, j}$ for which

$$
w_{1}^{p}\left(\lambda_{i, j}\right) w_{2}^{q}\left(\lambda_{i, j}\right)\left[M_{i, j}\right]=\left\{\begin{array}{lll}
0 & \text { if } q>j, & p+2 q=i+2 j \\
1 & \text { if } q=j, & p=i
\end{array}\right.
$$


Any collection of such manifolds form a base for $\mathfrak{N}_{*}\left(\mathrm{BO}_{2}\right)$ as $\mathfrak{N}_{*}$ module. The representatives will be chosen so that $\lambda_{i, j}$ is a subbundle of the tangent bundle of $M_{i, j}$ except for $j=0$ and $i \leqslant 3$.

For $j \geqslant 2$, one lets

$$
M_{i, j}=R P(1, \underbrace{0, \cdots, 0}_{j-1}) \times R P(1, \underbrace{0, \cdots, 0}_{i+j-1})
$$

and lets $\lambda_{i j}=\pi_{1}^{*}(\lambda) \oplus \pi_{2}^{*}(\lambda)$, where $\lambda$ is the canonical line bundle over $R P(1,0, \cdots, 0)$.

For $j=1, i \geqslant 3$, one lets $M_{i, j}=R P(1) \times R P(3,0, \cdots, 0)(i-20$ 's) and lets $\lambda_{i, j}=\pi_{1}^{*}(\xi) \oplus \pi_{2}^{*}(\lambda), \xi$ being the Hopf bundle over $R P(1)$. The tangent bundle of $R P(3)$ is trivial and so the tangent bundle of $M_{i, j}$ is $3 \oplus$ $\pi_{2}^{*}(\lambda) \otimes\left(\pi_{2}^{*}\left(\xi^{\prime}\right) \oplus(i-2)\right)$, where $\xi^{\prime}$ is the Hopf bundle over $R P(3)$. Since $2 \xi=2$ and $i \geqslant 3, \lambda_{i, j}$ is a subbundle of the tangent bundle.

For $j=0, i \geqslant 4$, one lets $M_{i, j}=R P(3,0, \cdots, 0)(i-30$ 's $)$ and $\lambda_{i, j}=1 \oplus \lambda$.

For $j=1, i=0$, one lets $M_{i, j}=R P(2)$, and $\lambda_{i, j}=\tau$, the tangent bundle of $R P(2)$.

For $j=1, i=1$, one lets $M_{i, j}=R P(1) \times R P(2)$, the tangent bundle being $1 \oplus \pi_{2}^{*}(\tau)=3 \pi_{2}^{*}(\xi)=\left(2 \pi_{1}^{*}(\xi) \otimes \pi_{2}^{*}(\xi)\right)+\pi_{2}^{*}(\xi)$ and lets $\lambda_{i, j}=\left[\pi_{1}^{*}(\xi) \otimes\right.$ $\left.\pi_{2}^{*}(\xi)\right] \oplus \pi_{2}^{*}(\xi)$.

For $j=1, i=2$, let $M_{i, j}$ be the bundle of lines in the fibers of $\lambda \oplus 2$ over $R P(1,0)=R P(\xi \oplus 1)$ where $\xi$ is the Hopf bundle over $R P(1)$, giving projections

$$
\pi: M_{i, J} \rightarrow R P(1,0), \quad p: R P(1,0) \rightarrow R P(1) .
$$

Let $\theta$ be the bundle along the fibers of $p, \eta$ the bundle along the fibers of $\pi$, and $\lambda^{\prime}$ the canonical line bundle over $M_{i, j^{\prime}}$. Then

$$
\tau_{M_{i, j}}=\eta \oplus \pi^{*}\left(\tau_{R P(1,0)}\right)=\eta \oplus \pi^{*}(\theta) \oplus 1=\left(\lambda^{\prime} \otimes \pi^{*}(\lambda \oplus 2)\right) \oplus \pi^{*}(\theta)
$$

which contains a copy of $\lambda_{i, j}=\lambda^{\prime} \oplus \pi^{*}(\theta)$.

Finally, let $M_{0,0}$ be a point with $\lambda_{0,0}$ trivial, $M_{1,0}=R P(1)$ with $\lambda_{1,0}=\xi \oplus 1$, and $M_{2,0}=R P(1,0), M_{3,0}=R P(1,0,0)$ with $\lambda_{i, 0}=\lambda \oplus 1$.

Note that for $M_{i, 0}, i \leqslant 3, \lambda_{i, 0}$ is a subbundle of $\tau \oplus 2$. In particular, if $\alpha \in \Re_{p}$ and $w_{p}(\alpha)=0, \alpha$ is represented by a manifold $M^{p}$ fibered over $S^{1} \times S^{1}$ [5, Proposition 6.1] and hence $\tau_{M}$ has 2 sections, so $\lambda_{i, 0}$ is a subbupdle of the tangent bundle of $M \times M_{i, 0}$.

Every class in $\mathfrak{N}_{n}\left(B O_{2}\right)$ is of the form $\Sigma \alpha_{(i, j)}\left[M_{i, j}, \lambda_{i, j}\right]$ with $\alpha_{(i, j)} \in$ $\mathfrak{N}_{n-i-2 j}$ and every class $\alpha \in \mathfrak{N}_{p}$ has the form $\beta+a R P(2)^{p / 2}, a \in Z_{2}, \beta \in \mathfrak{N}_{p}$ with $w_{p}(\beta)=0$. Thus if $I$ is the $\mathfrak{N}_{*}$ submodule of classes in $\mathfrak{N}_{*}\left(B O_{2}\right)$ 
represented by $[M, f]$ with $f^{*}\left(\gamma_{2}\right)$ a subbundle of the tangent bundle of $M$, then $\mathfrak{N}_{*}\left(B_{2}\right) / I$ is a $Z_{2}$ vector space generated by the classes

$$
\left[R P(2)^{s}\right] \cdot\left[M_{i, 0}, \lambda_{i, 0}\right] \quad \text { with } i \leqslant 3 \text {. }
$$

The characteristic numbers $w_{n}\left(\tau-f^{*}\left(\gamma_{2}\right)\right)$ and $w_{1}\left(\tau-f^{*}\left(\gamma_{2}\right)\right) \cdot$ $w_{n-1}\left(\tau-f^{*}\left(\gamma_{2}\right)\right.$ ) (for $\left.n \geqslant 2\right)$ may be readily seen to give a homomorphism $\Re_{n}\left(B O_{2}\right) / I \rightarrow Z_{2} \oplus Z_{2}$ (or to $Z_{2}$ if $n \leqslant 1$ ) sending the classes $\left[R P(2)^{s}\right] \cdot$ $\left[M_{i, 0} ; \lambda_{i, 0}\right]$ of dimension $n$ to linearly independent elements.

This completes the proof of the proposition.

\section{REFERENCES}

1. P. E. Conner and E. E. Floyd, Fibering within a cobordism class, Michigan Math. J. 12 (1965), 33-47. MR, 31 \#4038.

2. —, Torsion in SU.bordism, Mem. Amer. Math. Soc. No. 60 (1966). MR 32 \#6471.

3. R. Lashof, Poincaré duality and cobordism, Trans. Amer. Math. Soc. 109 (1963), 257-277. MR 27 \#6281.

4. R. E. Stong, Notes on cobordism theory, Princeton Univ. Press, Princeton, N. J.; Univ. of Tokyo Press, Tokyo, 1968. MR 40 \#2108.

5. - On fibering of cobordism classes, Trans. Amer. Math. Soc. 178 (1973), $431-447$.

DEPARTMENT OF MATHEMATICS, UNIVERSITY OF VIRGINIA, CHARLOTTESVILLE, VIRGINIA 22903 